\title{
Corrigendum to "Effects of Initial Porosity and Water Pressure on Seepage-Erosion Properties of Water Inrush in Completely Weathered Granite"
}

\author{
Jinquan Liu $\mathbb{D},{ }^{1,2}$ Weizhong Chen, ${ }^{3}$ Taogen Liu, ${ }^{4}$ Jianxin $Y u,{ }^{5}$ Jingliang Dong $\mathbb{D}^{1}{ }^{1}$ \\ and Wen Nie (iD $^{2}$ \\ ${ }^{1}$ School of Civil Engineering and Architecture, East China Jiaotong University, Nanchang, Jiangxi 330013, China \\ ${ }^{2}$ Quanzhou Institute of Equipment Manufacturing, Haixi Institutes, Chinese Academy of Sciences, Quanzhou, Fujian 362000, China \\ ${ }^{3}$ State Key Laboratory of Geomechanics and Geotechnical Engineering, Institute of Rock and Soil Mechanics, Chinese Academy \\ of Sciences, Wuhan, Hubei 430071, China \\ ${ }^{4}$ College of Civil Engineering \& Architecture, Nanchang Institute of Technology, Nanchang, Jiangxi 330013, China \\ ${ }^{5}$ School of Civil Engineering, Henan Polytechnic University, Jiaozuo, Henan 454000, China
}

Correspondence should be addressed to Jinquan Liu; jinquanliu99@163.com, Jingliang Dong; 429146390@qq.com, and Wen Nie; wen.nie@fjirsm.ac.cn

Received 24 September 2018; Accepted 24 September 2018; Published 29 October 2018

Copyright (c) 2018 Jinquan Liu et al. This is an open access article distributed under the Creative Commons Attribution License, which permits unrestricted use, distribution, and reproduction in any medium, provided the original work is properly cited.

In the article titled "Effects of Initial Porosity and Water Pressure on Seepage-Erosion Properties of Water Inrush in Completely Weathered Granite" [1], the fund number of the China National Natural Science Foundation was incorrect. The Acknowledgments section including the corrected fund number should be as follows:

"This work was supported by the China National Basic Research Program, the "973 Program" (no. 2013CB036006), and the China National Natural Science Foundation (nos. 51509246 and 51809253). These financial supports are gratefully acknowledged."

\section{References}

[1] J. Liu, W. Chen, T. Liu, J. Yu, J. Dong, and W. Nie, "Effects of initial porosity and water pressure on seepage-erosion properties of water inrush in completely weathered granite," Geofluids, vol. 2018, Article ID 4103645, 11 pages, 2018. 

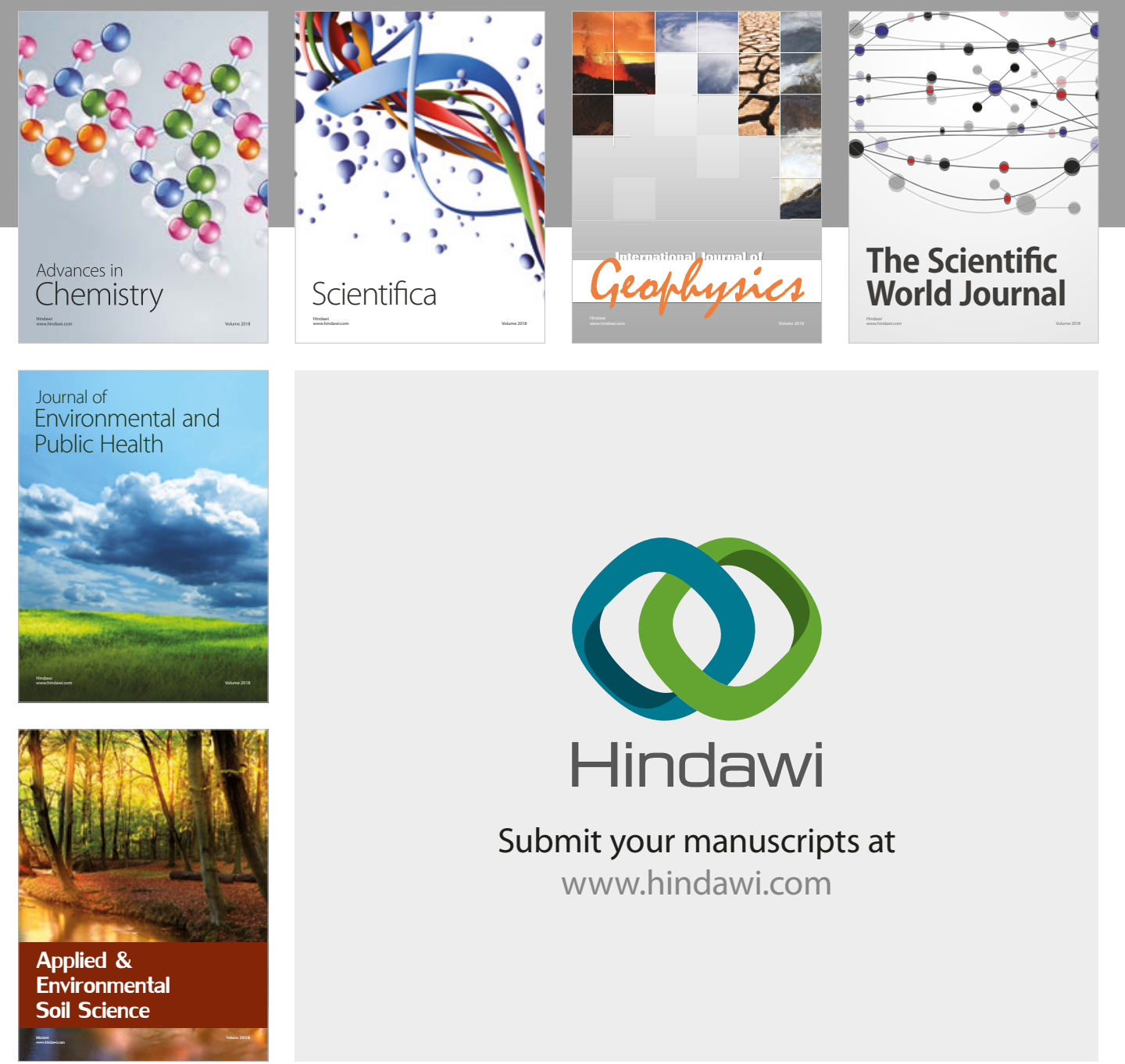

The Scientific

\section{World Journal}
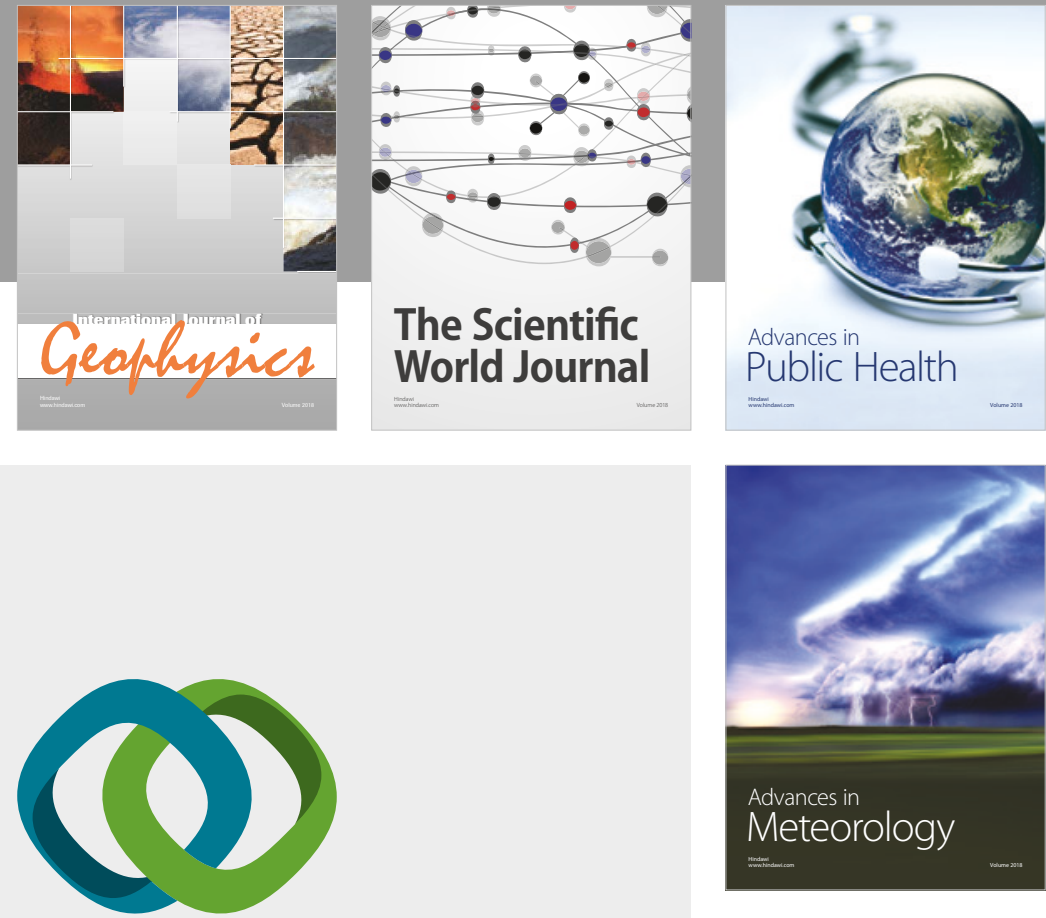

Advan

Public Health

\section{Hindawi}

Submit your manuscripts at

www.hindawi.com
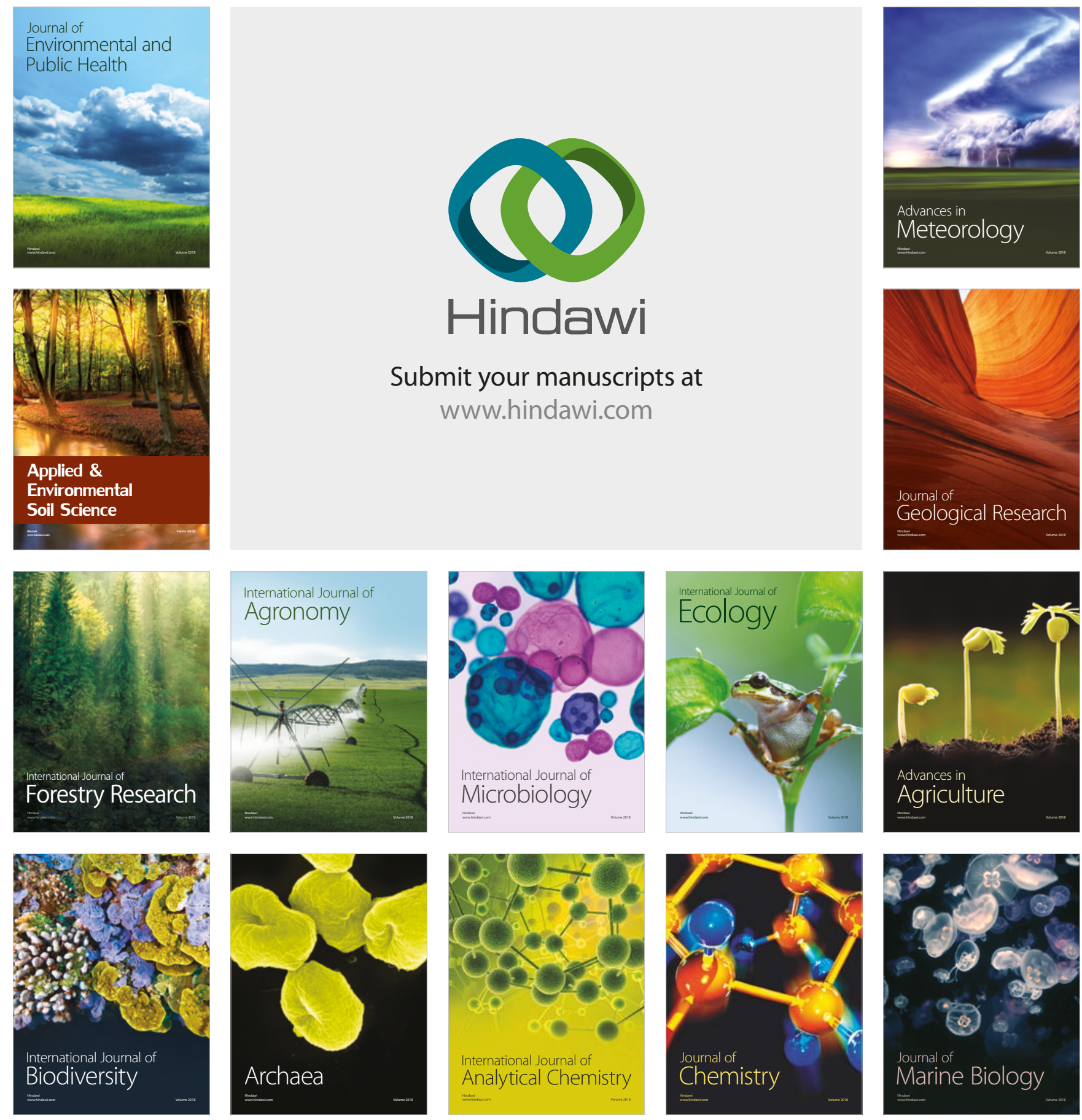